\title{
Ultrasons et Vis Instrumentées pour Sécuriser vos Assemblages Boulonnés
}

\author{
Jean-Philippe Godin - POLYMESURE, 92100 Boulogne-Billancourt, France
}

\begin{abstract}
La technologie proposée par la société AMG Intellifast permet de mesurer les efforts de serrage dans les assemblages vissés. Elle apporte des solutions concrètes pour maitriser la conception d'un produit, assurer sa validation pendant le développement et offrir une possibilité de maintenance préventive en vie courante. L'objet de cette présentation est de décrire la technologie mise en ouvre et de donner un aperçu des applications qui en bénéficient.
\end{abstract}

\section{Préambule}

En annexe C du document VDI2290 ou EN1591-1, le graphique montrant la dispersion des différentes méthodes de serrage sur le couple et sur la tension de serrage encourage l'utilisation de l'ultrason. Il peut être dans certains cas la seule solution pour la réduction de la dispersion sur la Tension d'Assemblage.

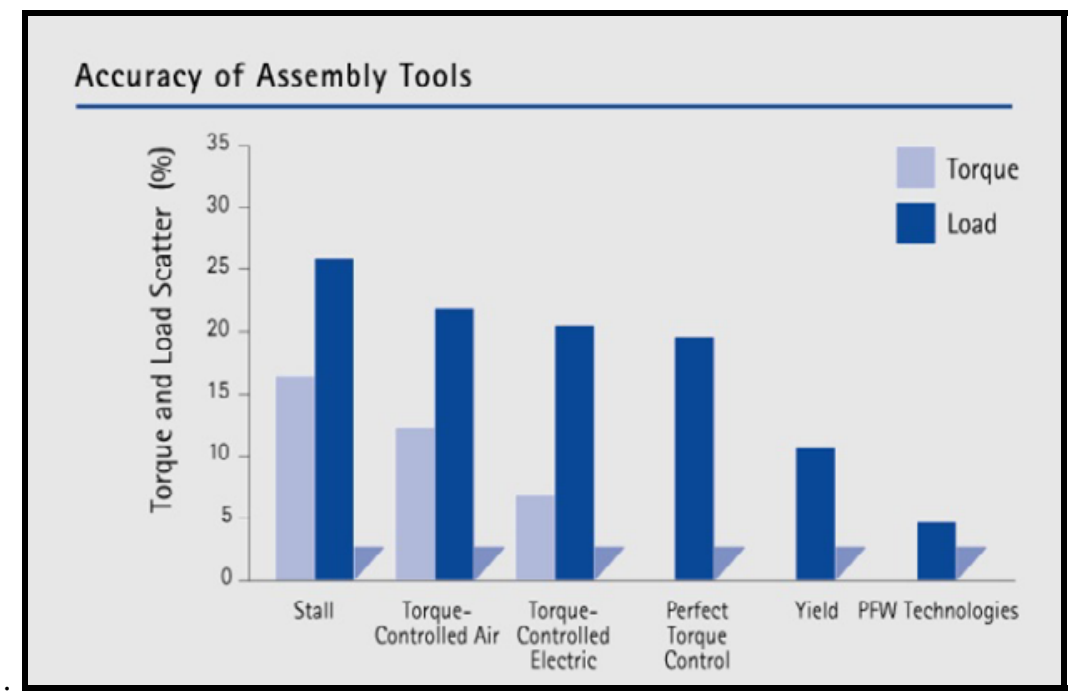

Figure 1. Précision des moyens d'assemblage

L'enjeu est important car cette méthode permet de réaliser le meilleur design tout en garantissant la sureté de fonctionnement de l'équipement. Cela peut donc avoir un impact positif en réduisant le coût engendré par une panne ou un casse (fuite, dépassement de la limite élastique, desserrage), ou même sur le coût de fabrication du produit (nombre et dimension des vis). 


\section{La mesure de serrage par ultrason}

La technologie de mesure de la Tension de serrage par Ultrason fait partie des nombreux moyens de mesure existants. D'un point de vue mécanique, la longueur des boulons augmente conformément à la loi de Hooke. Un effet plus significatif est la diminution de la vitesse du son au travers de la matière. Ainsi, en utilisant les ultrasons, il est possible de mesurer les effets conjugués de la réduction de leur vitesse de propagation et de l'augmentation de l'allongement du boulon. Ce qui conduit à mesurer un accroissement du temps de trajet de ces ultrasons sur lequel est appliquée une compensation liée aux effets de la température.

$\mathrm{Au}$ sein même de cette technologie, on retrouve plusieurs façons de générer les ultrasons permettant de mesurer la Tension de Serrage:

- Sonde de mesure et liquide couplant

- Capteur piézo céramique collé

- Capteur électro déposé de manière permanente sur la vis.

C'est cette dernière technologie qui est décrite plus précisément par la suite dans ce document.

\section{La technologie des capteurs permanents}

\subsection{Le procédé de fabrication}

La première étape consiste à préparer les boulons d'une manière mécanique et chimique. La géométrie et l'état de la surface sur laquelle le capteur va être déposé est prépondérante pour assurer la qualité du dépôt et des signaux qui seront générés.

De même, le retrait de toutes les impuretés ou des lubrificateurs présents sur les boulons est indispensable pour éviter toute pollution du four et des capteurs.

Ensuite, Les boulons sont disposés dans des grilles sur le pourtour de la chambre. Leur nombre peut atteindre quelques milliers en fonction de leurs dimensions.

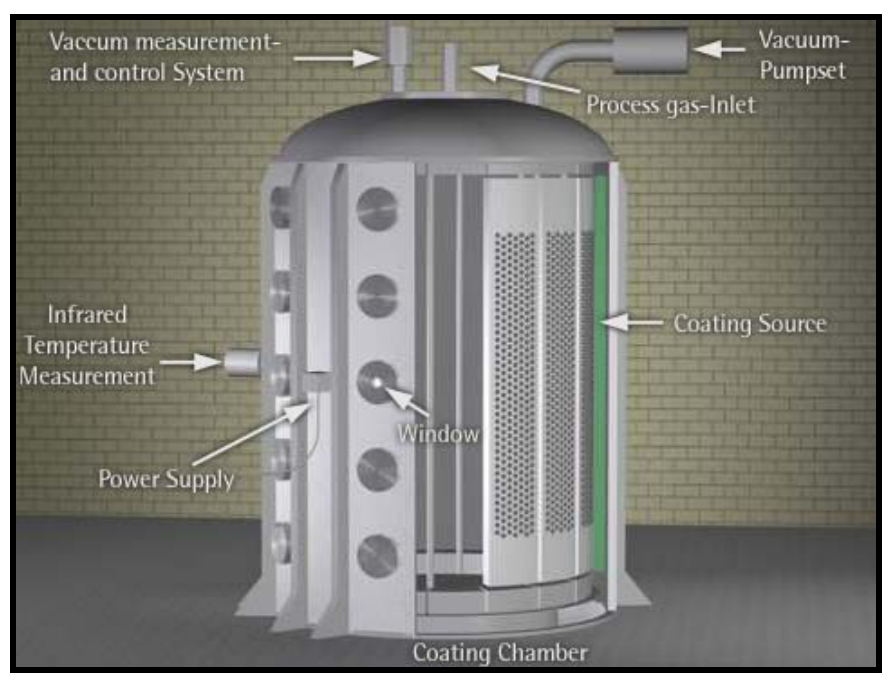

Figure 2. Four à électro déposition sous vide 
Les magnétrons installent un champ magnétique qui dirige les ions Argon sur des cibles solides. Celles-ci libèrent leurs ions sous forme de plasma et ceux-ci viennent se déposer sur la vis de manière permanente. Le capteur est déposé en 3 trois couches successives sur la surface de la tête de vis ou sur le pied de vis. Ces couches minces font quelques dizaines de microns d'épaisseur.

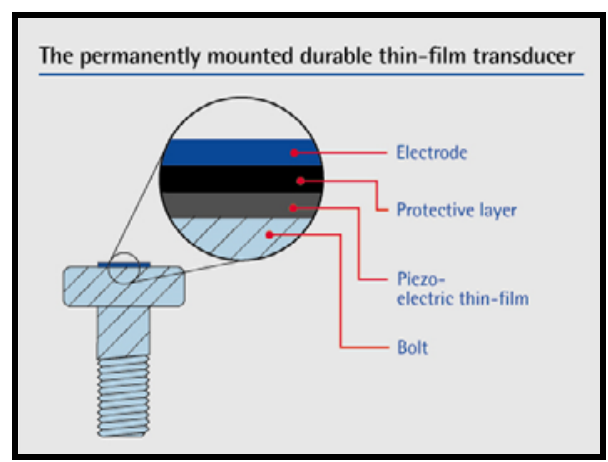

Figure 3. Composition du capteur permanent PMTS

La première couche est un piézo électrique, celui-ci est protégé par une 2ème couche. La 3ème couche est appliquée avec un masquage produisant ainsi deux zones qui permettront ensuite de créer un contact électrique pour activer le piézo. L'anneau peut être aisément situé sur le meilleur spot pour assurer des mesures répétables. Il est généralement concentrique avec l'axe des boulons.

Pour les boulons travaillant à des températures de $180^{\circ} \mathrm{C}$ maximum, la couche de protection est de l'étain. Pour des températures plus élevées, environ $320^{\circ} \mathrm{C}$, le revêtement est un composé Titane.

\subsection{Le principe de mesure}

Le piézo est comme un écho sondeur. Il agit à la fois comme un émetteur et comme un récepteur. Il émet un pulse d'énergie lorsqu'on lui applique une différence de potentiel. Ce pulse prend la forme d'une onde longitudinale et d'une onde transversale de fréquence comprise entre 6 et $25 \mathrm{MHz}$. Ces ondes sont réfléchies par le bout de la vis. Le temps que met l'impulsion à faire un aller et retour est donné en nanosecondes, ce qui permet une mesure très précise.

Le processus Intellifast permet de sélectionner le meilleur signal et ne prend en compte que le premier écho de l'onde longitudinale, celui-ci ayant l'amplitude la plus élevée.

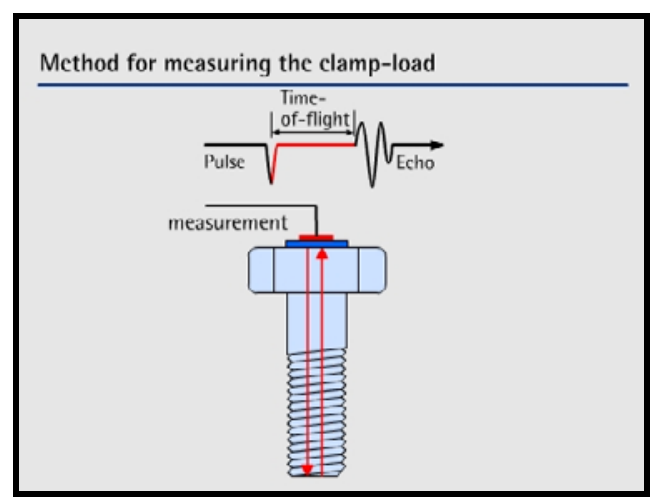

Figure 4. Principe de Mesure Pulse-Echo 
Un étalonnage permet de faire le lien entre les mesures de temps de vol en nanosecondes et la tension de serrage en $\mathrm{N}$ ou KN. Cette information sera donnée directement par l'instrumentation de mesure. La courbe d'étalonnage est issue d'une mesure faite avec des cellules d'effort sur le site d'Intellifast à Speyer mais également sur une machine de traction si la taille des boulons est trop importante pour être installé sur les cellules. Cet étalonnage donne généralement une précision de l'ordre de $+/-3 \%$. Pour des boulons spécifiques tels que de longs goujons ou des boulons d'ancrage où la flexion peut apparaitre, la précision peut s'approcher des $+/-5 \%$.

Pour des boulons dont le matériau n'est pas propice à la propagation des ondes, la précision peut être pénalisée car le signal retour risque d'être atténué et difficilement exploitable. Mais des solutions existent pour palier à ces problématiques comme l'étalonnage unitaire ou la reprise de la surface de réflexion pour concentrer les ondes vers l'axe central.

\subsection{Fonction additionnelle}

En complément de ces capteurs, AMG Intellifast propose une identification par code Datamatrix pour une meilleure traçabilité des mesures. Celui-ci est créé par évaporation sélective de la couche supérieure. Ce procédé permet donc de créer un code pratiquement inaltérable et utilisable sur toute la durée de vie de la vis.

Il contient les informations essentielles pour recharger automatiquement le numéro de la vis, la courbe d'étalonnage ainsi que son temps de vol de référence lorsqu'elle est libre. Après lecture du code, il ne reste plus qu'à disposer la sonde ultrason et la sonde de température sur le boulon pour effectuer la mesure.

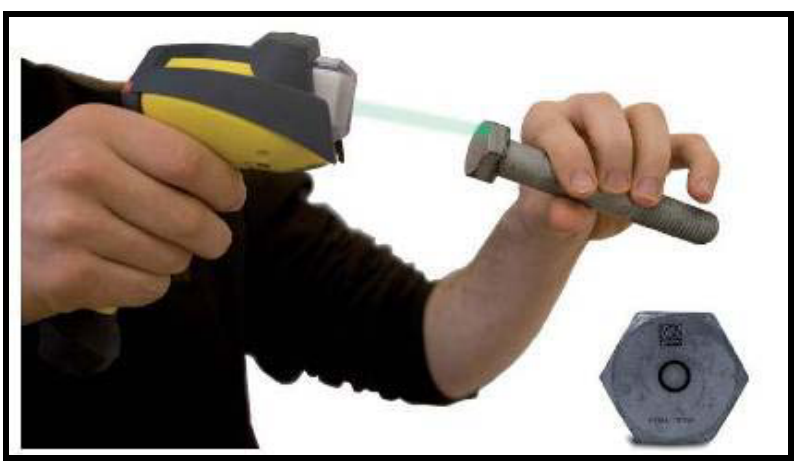

Figure 5. Traçabilité des boulons et des mesures par code Datamatrix

\section{Applications de la technologie}

L'apport de cette technologie au niveau des essais vibratoires en environnement est multiple et le système de mesure peut être décliné en fonction du besoin.

A l'installation du système à mesurer sur le banc d'essai, il peut s'avérer intéressant de maitriser les conditions aux limites au travers d'un serrage correct. Pour cela, le système LP Touch offre la possibilité de contrôler la Tension d'Assemblage par une mesure simple et rapide de chaque boulon. Les signaux analogiques sont récupérés depuis le traditionnel capteur ultrason placé sur la tête de vis et sont convertis en un signal numérisé. Les mesures sont visibles sur le LP Touch et également exportables pour l'intégration à un rapport d'essais. 


\subsection{Conception assemblage composite}

AMG Intellifast a eu l'occasion de collaborer avec la société GCT sur un exemple d'assemblage d'une structure composite. A partir d'un modèle de jonction composite assemblée par une vis, GCT a simulé les contraintes vue par les pièces en fonction de 2 méthodes de serrage : une au couple et l'autre à la tension de serrage. Deux hypothèses de frottement ont été considérées: 0,08 et 0,21 .

Pour un serrage au couple, ces frottements ont montré une dispersion d'environ $40 \%$ sur la tension d'assemblage alors qu'avec une méthode de serrage à la tension, on atteint des dispersions d'environ $5 \%$. Les calculs ont également montrés que les contraintes principales pouvaient être réduites dans l'assemblage lorsqu'on diminuait la dispersion de l'effort de serrage dans la vis.
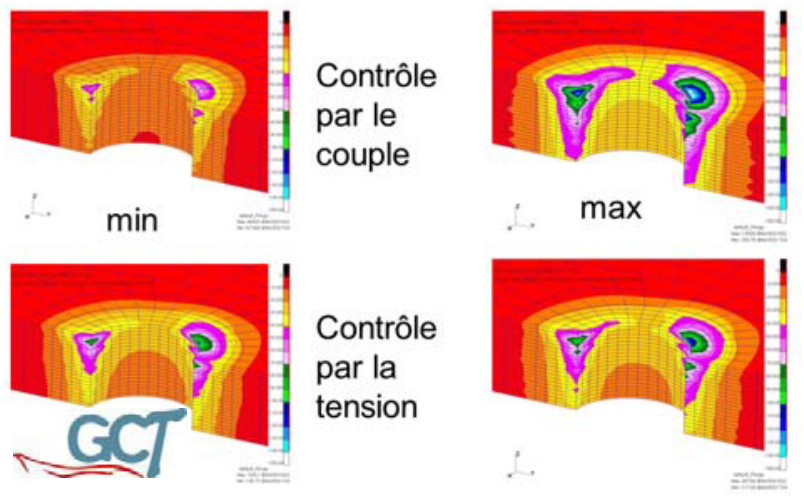

Figure 6. Maitrise des contraintes dans un assemblage composite

\subsection{Serrage à la Tension}

Si l'on veut effectuer un serrage au Couple tout en vérifiant la Tension installée, il est possible de connecter une clé dynamométrique spécialement équipée pour la mesure avec vis instrumentées et ultrasons. La clé E'Torc II est un équipement électronique qui permet de mesurer simultanément les 3 paramètres du serrage : le couple, l'angle et la Tension d'Assemblage.

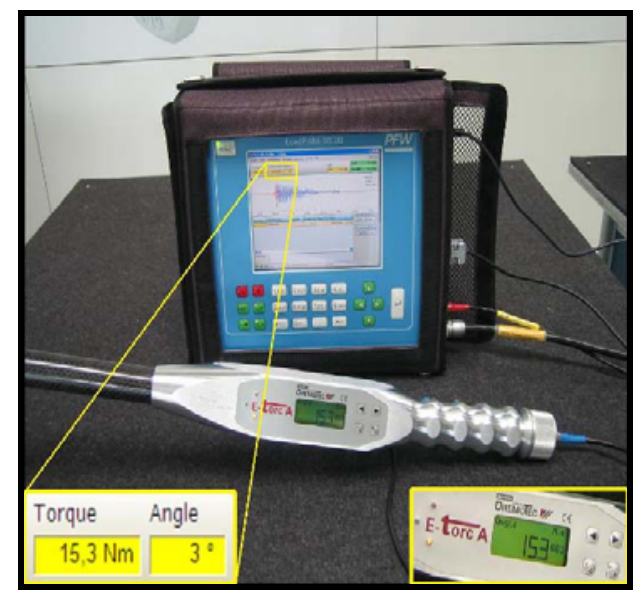

Figure 7. Clé dynamométrique pour serrage à la Tension 
Dans l'industrie, certaines applications ont nécessité l'utilisation de la clé associée à la technologie des capteurs permanents. L'exemple ci-dessous est tiré d'un essai réalisé chez Rolls-Royce Aerospace en charge du développement et de la fabrication de l'ensemble propulseur du Lockheed Martin F35B.

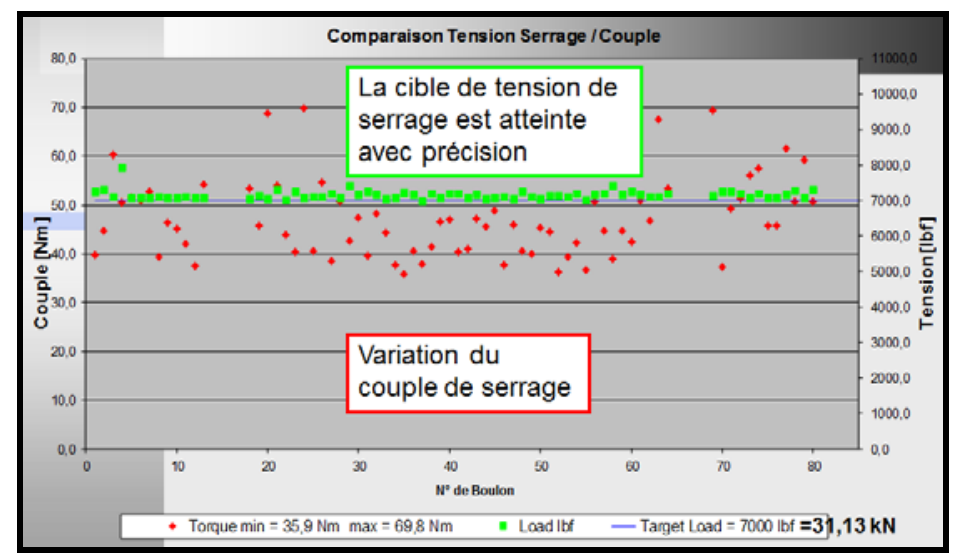

Figure 8. Comparaison serrage au couple et à la tension

Il apparait clairement qu'en visant une tension de serrage constante dans chaque assemblage (80 au total), on obtient une dispersion importante au niveau du couple en passant de $35 \mathrm{Nm}$ à $70 \mathrm{Nm}$. Si l'on raisonne à l'inverse, en effectuant des serrages à couple constant, on peut très bien imaginer les dispersions obtenues sur la tension de serrage avec les conséquences pour l'assemblage : desserrage des vis ou dépassement des limites élastiques avec risque de rupture.

\subsection{Mesure statique multivoie}

Pendant les phases de développement d'un produit, la validation de ses fonctions et de sa tenue vis à vis de contraintes externes est à vérifier.

Dans ce cadre, un client a expérimenté le monitoring des boulons d'un rotor grâce aux vis instrumentées. L'objectif principal était de réaliser les essais de fatigue du rotor en évitant d'arrêter le banc sur casse d'un élément annexe. C'est pourquoi ce client a souhaité suivre en temps réel la tension de serrage statique de plusieurs boulons du rotor afin de prévenir leur rupture.

Il a donc mis en œuvre un système qui pouvait s'apparenter à celui présenté dans la figure ciaprès : un ensemble d'acquisition monovoie formé par le PC et le boitier SCMU ; un multiplexeur pour connecter le système à plusieurs boulons et permettent de réaliser le contrôle en séquentiel.

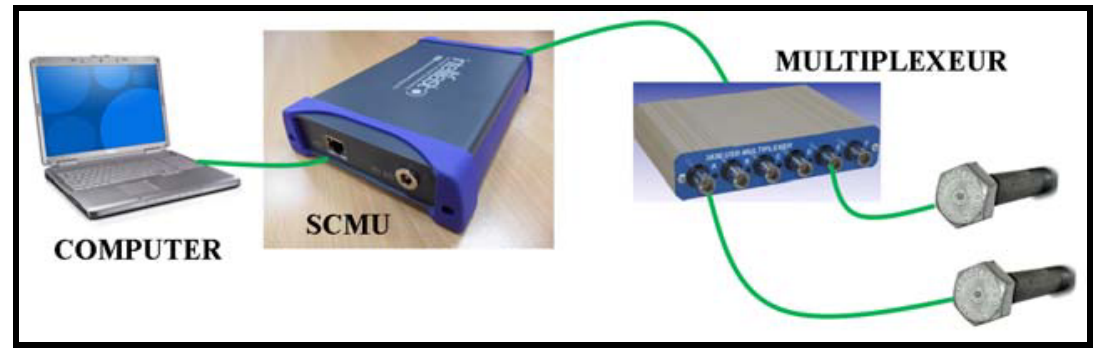

Figure 9. Système de monitoring mono voie 
En complément de cet exemple d'application, on peut également citer:

- Le suivi de l'effort statique de maintien des pièces en fonction des vibrations et de la température par des mesures périodiques des valeurs statiques $\Rightarrow$ vérification de la relaxation de l'assemblage, du desserrage ou du risque de casse

- Le suivi de la tension des boulons qui maintiennent une machine sur un châssis lors du transport par camion $\Rightarrow$ possibilité d'ajuster le serrage pour éviter la perte de boulons ou un mauvais serrage engendrant des vibrations dommageables pour le produit

- La maintenance préventive à distance des boulons d'une éolienne ou plus généralement d'une machine $\Rightarrow$ optimisation des périodes de contrôle sur site et des déplacements à organiser.

\subsection{Mesure dynamique multivoie}

Le deuxième système décrit ci-après est plus orienté vers la mesure dynamique synchronisée de plusieurs boulons. Il est constitué d'un ou plusieurs racks qui comportent jusqu'à 8 cartes d'acquisition, une carte par boulon. Cet ensemble de racks peut être disposé dans une armoire localisée à distance des boulons à mesurer. Les cartes sont reliées à un switch qui renvoi par un seul câble Ethernet l'ensemble des mesures vers un PC équipé d'un logiciel spécifique.

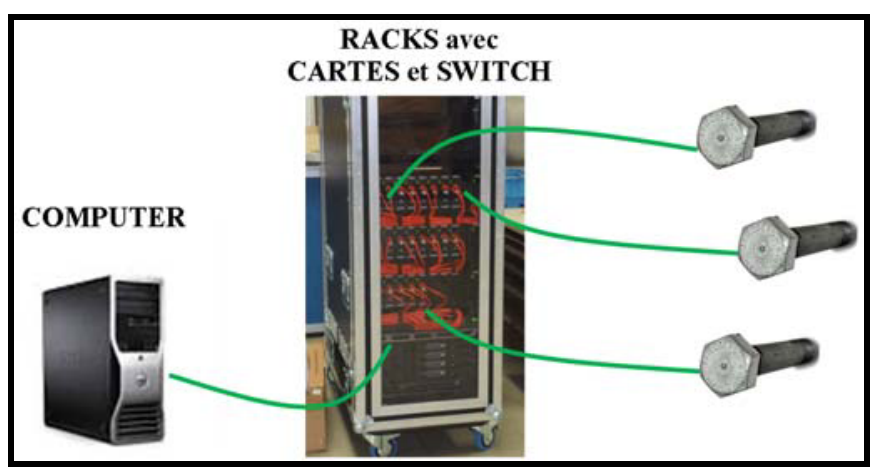

Figure 10. Système de monitoring multi voie

Ici nous sommes plutôt dans le cadre de tests dynamiques où est réalisée l'analyse du comportement en fréquence des boulons. Les vis instrumentées remplacent avantageusement les cellules d'effort car elles sont non intrusives : elles ne modifient pas la longueur assemblée, un des éléments essentiels impactant le comportement de l'ensemble boulonné.

Sur deux exemples d'application «client», nous avons mis en œuvre un système comportant 21 voies de mesure et fonctionnant à une fréquence d'acquisition de $10 \mathrm{~Hz}$ pour le premier, et un système avec 8 voies de mesure et une fréquence d'échantillonnage de l'ordre de $340 \mathrm{~Hz}$ pour le second.

Les développements récents de nouvelles cartes d'acquisition et de logiciels performants ont permis d'atteindre des fréquences d'échantillonnage de $1000 \mathrm{~Hz}$ pour un maximum de 10 voies de mesure, moyennant un ordinateur puissant pour supporter la vitesse d'échange des données. 


\section{Conclusion}

L'assemblage boulonné reste un classique des assemblages mécaniques. Il a fait l'objet de nombreuses études et sa maitrise est assurée par l'expérience acquise. Cependant, comme dans toute technique, des avancées sont encore possibles pour accroitre la connaissance des assemblages et optimiser leur conception afin de répondre aux défis actuels et futurs. D'autant plus que le contexte technique et économique peut amener à faire des choix qui remettent en cause l'intégrité d'un assemblage.

C'est pourquoi, afin d'appuyer les équipes qui se penchent sur les problématiques de serrage et font face à des évolutions techniques qui poussent plus loin la tenue des assemblages, il nous semble intéressant de proposer la solution technologique des vis instrumentées associée à la mesure par ultrason.

Elle peut s'avérer une aide précieuse à toutes les étapes de la vie d'un produit : dès la conception afin d'optimiser le design, pour la validation d'un concept ou lorsque la définition finale est figée, pour le contrôle du serrage sur chaine de production et enfin pendant les phases de maintenance en vie courante. 